\title{
Failure of "effective" treatment for heart failure to improve normal customary activity
}

\author{
John T Walsh, Richard Andrews, Alison Evans, Alan J Cowley
}

\begin{abstract}
Objectives-To examine the effects of drug treatment on laboratory exercise tests in relation to measures of daily activity in patients with chronic heart failure.

Setting-University teaching hospital. Subjects-18 patients with mild to moderate chronic heart failure (New York Heart Association functional class II-III) and 10 age matched healthy controls. Methods-Assessments were made before and after 12 weeks of vasodilator drug treatment. Exercise capacity was measured during two different types of treadmill exercise, one using a ramp protocol and the other a fixed work load. Corridor walk tests at three self selected speeds were also undertaken and measures of customary activity assessed from pedometer scores.
\end{abstract}

Results-Exercise times were significantly increased from baseline $(P<0.01)$ with both treadmill protocols after 12 weeks of drug treatment, with a positive correlation between the duration of treadmill exercise for both protocols $(r=0.69, P<0.01)$. Corridor walk tests of $100 \mathrm{~m}$ at a self selected slow speed also improved $(P<0.02)$ but these did not correlate with the changes in treadmill exercise time. The pedometer scores of the patients with heart failure were greatly reduced compared with those of the controls $\left(258(45) \times 10^{2} v 619(67) \times\right.$ $10^{2}$ steps/week, $\left.P<0.001\right)$ and after 12 weeks of treatment were unchanged (261 (42) $\times 10^{2}$ steps/week).

Conclusions-These data confirm the need to use different exercise protocols when assessing the benefits of drug treatment in patients with chronic heart failure. Treatments that seem effective with conventional laboratory based exercise tests may not improve daily activities. This may reflect a failure of apparently successful treatment and should be considered when intepreting clinical trials.

Medicine, University

Hospital, Nottingham

J T Walsh

$\mathrm{R}$ Andrews

A Evans

A J Cowley

Correspondence to:

Dr J T Walsh,

Cardiovascular Medicine,

University Hospital,

Nottingham NG7 2UH

Accepted for publication

11 April 1995

(Br Heart f 1995;70:373-376)

Keywords: chronic heart failure; exercise protocols; exercise capacity; effects of drug treatment

An improvement in symptomatic wellbeing is an important aim in the treatment of patients with chronic heart failure but the best way of assessing it is not clear. Formerly, favourable changes in central haemodynamic variables were believed to indicate a beneficial effect of treatment but it is now recognised that they may not be matched by changes in the patient's exercise capability. ${ }^{12}$ As exercise capability is thought to be closely related to wellbeing, laboratory based, symptom limited exercise tests performed on a treadmill or bicycle are the usual way of assessing the effects of drugs. These tests have the advantage of close control but clearly are not representative of the type of exercise that patients perform during normal daily life. ${ }^{3}$ Different exercise protocols also seem to give different measures of incapacity and which is the most appropriate is not known. ${ }^{4}$

For these reasons other tests, such as corridor walk tests, in which patients decide their own work load are being used more frequently. These are of two types: those that are also symptom limited, patients walking as far as possible in an allotted time, ${ }^{5}$ and those that measure perception of speed in which patients are asked to walk a fixed distance at self selected speeds. ${ }^{6}$ Although these tests are more likely to be representative of daily incapacity, they remain laboratory based and as such may still not accurately reflect actual exercise capability outside of this environment.

Assessment of patients outside the laboratory often involves the use of quality of life questionnaires, many of which specifically enquire about levels of daily activity. Additional information may also be obtained using body borne pedometers, which have shown a greatly reduced level of daily activity in patients with heart failure compared with that in normal controls. ${ }^{4}$ Ideally, an effective drug should be able to increase this aspect of a patient's exercise capability and produce a real improvement in wellbeing. We have analysed results from patients enrolled in ongoing clinical trials to investigate whether an increase in exercise tolerance in laboratory based tests is paralleled by an improvement in daily customary activities.
Patients and methods

PATIENTS

Eighteen patients with moderate to severe heart failure were studied. They were participating in two trials that compared the effects of captopril and the oral dopamine agonist ibopamine, and isosorbide mononitrate and the angiotensin converting enzyme inhibitor, 
trandolapril. The patients' ages ranged from 56 to 75 years and 14 were men. The cause of heart failure was ischaemic heart disease in 10 patients, mitral or aortic regurgitation with impaired left ventricular function in four, and dilated cardiomyopathy in the remaining four. All patients were limited on exertion by symptoms of heart failure despite at least $80 \mathrm{mg}$ of frusemide daily (mean (range) dose was 90 $(80-160) \mathrm{mg}$ ). The studies were approved by the local ethics committee and all patients gave informed written consent.

Customary activity of 10 fit healthy controls of the same age as the patients was measured over a one week period.

\section{METHODS}

All patients were evaluated in an identical way. In addition to their normal medication they were given, single blind, placebo treatment. This was continued until their treadmill exercise time measured at weekly intervals using a modified Bruce protocol had reached a plateau and did not increase by more than $5 \%$ on two consecutive visits. Patients were then randomised to one of the active treatments and repeat measurements of exercise capacity were made at the end of 12 weeks of treatment.

\section{TREADMILL EXERCISE TESTS}

At each visit the patients were asked to exercise to a symptom limited maximum using two different treadmill protocols. The first was a modified Bruce protocol; the speed and slope of the treadmill increasing after three min at each of the following stages:

$\begin{array}{lllllll}\text { Stage } & \text { I } & \text { II } & \text { III } & \text { IV } & \text { V } & \text { VI } \\ \text { Speed }(\mathrm{km} / \mathrm{h}) & 2 \cdot 7 & 2 \cdot 7 & 2 \cdot 7 & 2 \cdot 7 & 4 \cdot 0 & 5 \cdot 4 \\ \text { Slope }^{\circ} & 0 & 1 \cdot 3 & 2 \cdot 6 & 4 \cdot 3 & 5 \cdot 4 & 6 \cdot 3\end{array}$

After at least $45 \mathrm{~min}$ of rest the patients were asked to complete a second symptom limited test. The speed and slope of the treadmill were fixed at the equivalent to stage IV of the modified Bruce protocol and patients continued at this work load until stopped by symptoms of heart failure.

Absolute work completed was calculated in kilojoules from standard formulae during both exercise tests.

\section{CORRIDOR WALK TESTS}

The patients rested for a further $30 \mathrm{mins}$ after the second treadmill exercise test, and were then asked to walk a $100 \mathrm{~m}$ along a corridor at self selected slow, normal, and fast speeds. The time to complete the $100 \mathrm{~m}$ walk at these speeds was recorded.

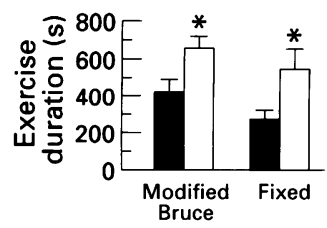

Figure 1 Effects of drug treatment on treadmill exercise duration. Values are mean (SEM). Before treatment; $\square$, after Before treatment; ${ }^{\star} P<0.01$, treatment. ${ }^{\star} P<0.01$,
Wilcoxon signed rank test.
CUSTOMARY ACTIVITY (PEDOMETER SCORES)

At all times during the study the patients wore body borne pedometers to evaluate their physical activity during normal daily activities measured by the total number of steps walked. The methods and pedometers have been validated previously. ${ }^{8}$ The scores were recorded at weekly intervals and the pedometers were calibrated at each visit. Pedometers were worn on belts, one at each hip. Scores were accepted only if the readings from both pedometers were within $20 \%$ of each other and a mean score was then calculated.

\section{STATISTICAL ANALYSIS}

Data are expressed as means (SEM). Analysis of the differences between variables before and after treatment was made with the Wilcoxon signed rank test. Analysis of the relations between the changes of the different variables was performed with Spearman's rank correlation coefficient.

\section{Results}

TREADMILL EXERCISE TESTS

The mean (SEM) symptom limited exercise time increased with both treadmill protocols (fig 1). Using the modified Bruce protocol exercise duration increased from $414(70) \mathrm{s}$ to 651 (68) s $(P<0.01)$. The baseline exercise duration was shorter with the fixed protocol $(P<0.01)$ but this also increased with treatment from $267(50) \mathrm{s}$ to $541(106) \mathrm{s} \quad(\mathrm{P}<$ $0.01)$. Figure 2 shows the correlation of the changes between the two treadmill protocols after treatment $(r=0.69, \mathrm{P}<0.05)$.

The mean (SEM) work performed also increased from $5.81(1.75) \mathrm{kJ}$ to $13.98(2.93)$ $\mathrm{kJ}(\mathrm{P}<0.01)$ using the modified Bruce protocol, and from $11.88(2.43) \mathrm{kJ}$ to $34.09(10.9)$ $\mathrm{kJ}(\mathrm{P}<0.01)$ with the fixed protocol. The baseline exercise capability assessed by total work was greater with the fixed protocol than the Bruce protocol $(P<0.01)$. There was a significant correlation between the protocols in respect of work completed $(r=0.5, \mathrm{P}<$ $0 \cdot 05)$.

\section{CORRIDOR WALK TESTS}

The mean (SEM) time taken for patients with heart failure to walk $100 \mathrm{~m}$ at a self paced slow speed decreased from 109 (6) s to 102 (6) s $(P<0.02)$ after treatment. Treatment had no significant effect at the normal (from 87 (4) s to 85 (5) s) and fast speeds (from 74 $(0.2) \mathrm{s}$ to $73(4) \mathrm{s})$. There were no significant correlations between the corridor walk tests and any aspect of treadmill exercise tolerance (table).

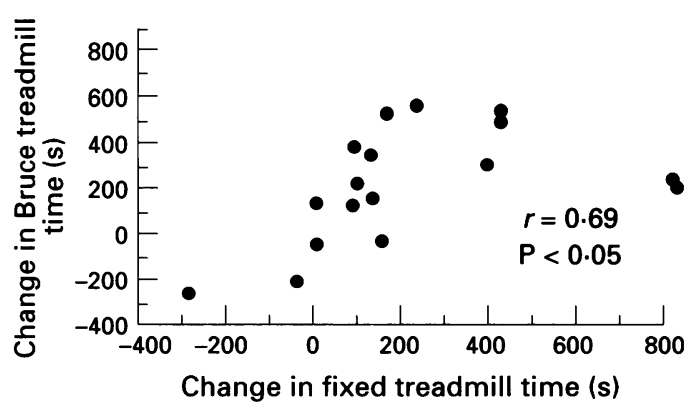

Figure 2 Pairwise correlation of changes in treadmill exercise capacity. 
Pairwise correlation of the change from baseline between different exercise tests

\begin{tabular}{|c|c|c|c|}
\hline & $\begin{array}{l}\text { No of } \\
\text { patients }\end{array}$ & $\begin{array}{l}\text { Correlation } \\
\text { coefficient }\end{array}$ & $\begin{array}{l}P \\
\text { value }\end{array}$ \\
\hline $\begin{array}{l}\text { Treatmill time (Bruce) } \\
v \text { fixed treadmill time } \\
v \text { slow walk } \\
v \text { normal walk } \\
v \text { fast walk } \\
v \text { pedometer score }\end{array}$ & $\begin{array}{l}18 \\
18 \\
18 \\
18 \\
18\end{array}$ & $\begin{array}{r}0.69 \\
-0.17 \\
-0.08 \\
-0.36 \\
-0.16\end{array}$ & $\begin{array}{l}0.02 \\
0 \cdot 25 \\
0.37 \\
0.07 \\
0 \cdot 26\end{array}$ \\
\hline $\begin{array}{l}\text { Treadmill time (fixed) } \\
v \text { slow walk } \\
v \text { normal walk } \\
v \text { fast walk } \\
v \text { pedometer score }\end{array}$ & $\begin{array}{l}18 \\
18 \\
18 \\
18\end{array}$ & $\begin{array}{l}-0.8 \\
-0.35 \\
-0 \cdot 26 \\
0.21\end{array}$ & $\begin{array}{l}0 \cdot 13 \\
0 \cdot 08 \\
0 \cdot 15 \\
0 \cdot 21\end{array}$ \\
\hline $\begin{array}{l}\text { Walk test } \mathrm{v} \text { pedometer score } \\
\text { Slow } \\
\text { Normal } \\
\text { Fast }\end{array}$ & $\begin{array}{l}18 \\
18 \\
18\end{array}$ & $\begin{array}{l}0.14 \\
0.08 \\
0.29\end{array}$ & $\begin{array}{l}0 \cdot 28 \\
0.37 \\
0 \cdot 12\end{array}$ \\
\hline
\end{tabular}

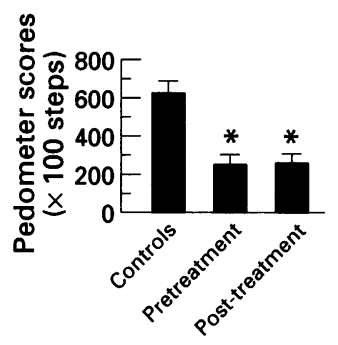

Figure 3 Pedometer scores of patients with heart failure before and after treatment. Values are means (SEM).

$\star P<0.001$ v controls,

Wilcoxon signed rank test.
CUSTOMARY ACTIVITY (PEDOMETER SCORES) The mean (SEM) pedometer score for normal controls was $619(67) \times 10^{2}$ steps. The mean (SEM) pedometer score for patients with heart failure was $258(45) \times 10^{2}$ steps over a one week period before treatment $(P<$ $0.001)$. There was no change after 12 weeks of vasodilators, the mean score being 261 (42) $\times 10^{2}$ steps (fig 3 ). There was no correlation between the changes in pedometer scores and any other aspect of exercise tolerance either on the treadmill or during the self paced corridor walk tests (table).

\section{Discussion}

Although improvement in the quality of life is a major aim of treatment of patients with chronic heart failure, this is not always addressed in clinical trials. Quality of life questionnaires are increasingly used to provide a subjective assessment of symptomatic impairment and response to treatment yet objective measures of exercise capability are frequently used to quantify this, usually in the laboratory on a bicycle or treadmill. Such tests allow close supervision but because protocols have fixed changes in work load the exercise performed is not normal and exercising to a symptom limited maximum is an unusual type of exercise. Furthermore, the most appropriate of the many available protocols is not known. ${ }^{34}$ For these reasons different measures of exercise capability that relate more closely to a patient's incapacity during normal daily activities would be advantageous. Corridor walk tests are self paced and therefore likely to be a better indicator of incapacity but as they are also conducted under supervision in an institution they may have some of the drawbacks of treadmill tests. A measure of incapacity obtained during normal daily activity should be the best indicator of symptomatic impairment but how it relates to other methods for assessing exercise tolerance is unknown.

In this study patients seemed to have different levels of exercise tolerance when measured on a treadmill with different protocols and depending on the way in which the results were expressed. If exercise time is used as the measure of capability then patients seemed less incapacitated with the Bruce protocol than the fixed work load protocol, while if the results are expressed as total work performed they are more incapacitated with the Bruce protocol. There was no relation between either exercise test and any other of the methods that we used to measure exercise capability so the different tests are measuring different aspects of patient's incapacity. We have also shown that patients had a pronounced reduction in customary activity measured with the pedometers compared with that of the healthy controls.

All patients received active treatment and after 12 weeks' treatment exercise tolerance was improved when assessed on the treadmill. It seems likely that such a significant increase reflects a true response to active treatment rather than a placebo effect, although a placebo control group was not included in the study. There was also a correlation between the changes with both protocols but the magnitude of the changes differed according to the protocol used. This clearly indicates that the measurement of incapacity and the response to treatment are dependent on the exercise protocol used. The treatments also improved the corridor walk test at the self selected slow pace with no significant effect at normal or fast speeds. This is similar to other studies that have reported improvement only at the slow speed. ${ }^{46}$ There was no correlation between the changes in corridor walk tests and the other tests.

Despite the clear improvement in the laboratory based tests surprisingly there was no change in customary activity measured with the pedometers after treatment. At baseline the patients had a profound reduction in customary activity compared with that of the controls and there was considerable room for improvement. The reason for the lack of improvement is not obvious. Patients may have already adapted their lifestyle to their reduced exercise ability and despite symptomatic improvement did not wish to increase daily activities. Alternatively, the pedometers measure only total steps and do not provide information about speed of walking or rest periods and it may thus be possible that while patients did not increase their total activity they exercised in more comfort with fewer rests.

The results from this study emphasise the need to use different exercise protocols to measure the response of patients with chronic heart failure to treatment. Although symptom limited exercise tests are useful in determining prognosis, tests most representative of a patient's normal daily activities should be used to assess the efficacy of drugs. It is not possible to conclude whether the lack of change in daily activity truly reflects a failed therapeutic response and quality of life assessments may have proved useful in this regard. Measures of customary activity in patients with chronic heart failure has, however, 
revealed a failure of apparently "effective" treatment in patients with a laboratory treatment response.

1 Franciosca JA, Goldsmith SR, Cohn JN. Contrasting immediate and long term effects of isosorbide dinitrate Med 1980;69:559-66.

2 Massie BM, Krammer BL, Topic N. Lack of correlation between short term haemodynamic effects of captopril between short term haemodynamic effects of captopril
and subsequent clinical responses. Circulation 1984;69:
$1135-41$.

3 Francis GS, Rector TS. Maximal exercise tolerance as a therapeutic end point in heart failure-are we relying on the right measure? Am $\mathcal{F}$ Cardiol 1994;73:304-6.
4 Cowley AJ, Fulwood L, Stainer K, Hampton JR. Exercise tolerance in patients with heart failure-how should it be measured? Eur Heart $\mathcal{f}$ 1991;12:50-4

5 Lipkin DP, Scriven AJ, Crake T, Poole-Wilson PA. Six minute walk test for assessing exercise capacity in patients with chronic heart failure. $B M \mathcal{F} 1986 ; 292$ 635-7.

6 Cowley AJ, Stainer K, Wynne RD, Rowley JM, Hampton JR. Symptomatic assessment of patients with heart failure: double blind comparison of increasing doses of diuretics and captopril in moderate heart failure. Lancet 1986;ii: $770-2$.

7 Hoodless DJ, Stainer K, Savic N, Batin P, Hawkins M Cowley AJ. Reduced customary activity in chronic heart failure: assessment with a new shoe mounted pedometer. Int $\mathcal{F}$ Cardiol 1994;43:39-42.

8 Bassey EJ, Dallosso HM, Fentem PH, Irving JM, Patrick JM. Validation of a simple mechanical accelerometer (pedometer) for the estimation of walking activity. Eur $\mathcal{F}$ Appl Physiol 1987;56:323-30. 
before any clinical component of their course; recent recommendations that all applicants to medical school should be screened, and immunised if appropriate, have far-reaching implications and have provoked controversy. ${ }^{17} 18$

In conclusion, among invasive cardiologists in the United Kingdom there is clear scope for improvement in vaccine uptake, particularly in completion of the immunisation regimen. Screening of patients, as recommended, should also be performed. Careful practice to avoid needle stick injury should continue and immunisation should also be offered to nursing and other ancillary staff caring for patients undergoing invasive cardiac procedures.

We thank Dr J Heptonstall, Consultant Medical Microbiologist, Public Health Laboratory Service Communicable Disease Surveillance Centre, London for invaluable advice before the preparation of this report. We also thank all those who completed and returned the questionnaire.

1 Recommendations of the Advisory Group on Hepatitis Protecting health care workers and patients from hepatitis Protecting health care work

2 Zuckermann A. Who should be immunised against hepatitis B? BMF 1984;289:1243

3 Recommendations of the Expert Advisory Group on AIDS Guidance for clinical health care workers: protection against infection with HIV/hepatitis B. HMSO, London 1990.

\section{CORRECTIONS}

Failure of "effective" treatment for heart failure to improve normal customary activity. $\mathcal{F} T \mathrm{Walsh}, R$ Andrews, $A$ Evans, $A \mathcal{F}$ Cowley (Br Heart $\mathcal{f}$ 1995;74: 373-6).

Exercise ventilation after balloon dilatation of the mitral valve. A P Banning, N P Lewis, $\mathcal{f} S$ Elborn, $R \mathcal{F}$ $C$ Hall (Br Heart $\mathcal{F} 1995 ; 74: 386-9)$.

We regret that the incorrect volume number was given in the strap lines and at the foot of the abstracts of these papers. The correct volume number is 74

Permanent pacemaker practice at a Scottish district general hospital between 1987 and 1994. $\mathcal{F} G$ Doherty, F Dawson, F Kerr

We regret that an error appeared in the second sentence of the discussion on page 477 of this article $(\mathrm{Br}$ Heart $\mathcal{F}$ 1995;73:475-8) which should have read, "Our average implantation rate over the 79 months $(130 / \mathrm{mil}$ lion population/year) compares with the United Kingdom national 1989 value of 148 /million population/year." $"$
4 Centers for Disease Control guidelines for prevention of HIV and hepatitis B viruses to health care and public safety workers. $M M W R$ 1989;38(suppl 56, 4-5):31-3.

5 Department of Health. Immunisation against infectious diseases. London: HMSO, 1992:110-9.

6 Polakoff S. Acute viral hepatitis B, reported to the Public Health Laboratory Service. F Infect 1990;20:163-8.

7 Heptonstall J. Outbreaks of hepatitis B virus infection associated with infected surgical staff. Communicable Disease Report 1991;1(8):R81-5.

8 Cartwright A. Professionals as poor responders: variations in and effects of response rates to questionnaires 1961-77. BMF 1978;ii:1419-21.

9 Editorial. Few physicians vaccinated against hepatitis B. Wis Med $\mathcal{F} 1989 ; 88: 14$.

10 Kinnersley P. Attitudes of general practitioners towards their vaccination against hepatitis B. BMf 1990;300:238.

11 Burden AD, Whorwell PJ. Poor uptake of hepatitis B immunisation amongst hospital-based health care staff. Postgrad Med F 1991;67:256-8.

12 Buss PW, McCabe M, Verrier Jones ER. Attitudes of paediatricians to HIV and hepatitis B virus infection. Arch Dis Child 1991;66:961-5.

13 Berridge DC, Galea MH, Evans DF, Pugh S, Hopkinson BR, Makin GS. Hepatitis B immunisation in vascula surgeons. Br f Surg 1990;77:585-6.

14 Williams JR, Flowerdew ADS. Uptake of immunisation against hepatitis $B$ among surgeons in Wessex Regional Health Authority. BMF 1990;301:154.

15 Le F, Porteous MJ. Operating practices of and precautions taken by orthopaedic surgeons to avoid infection with HIV and hepatitis B virus during surgery. BMF 1990; 301:167-9.

16 Lever AML. Treatment of the chronic hepatitis B virus carrier state. $\mathcal{F}$ Infect 1988;16:221-9.

17 Lever AML. Editorial: Hepatitis B and medical studen admission. $B M F$ 1994;308:870-1.

8 Kingman S. Hepatitis B status must be known for medical school. $B M \Im$ 1994;308:876.

The 1996 Annual General Meeting of the British Cardiac Society will take place at the Scottish Exhibition \& Conference Centre, Glasgow from 7 to 9 May.

The Fourth Annual Molecular Symposium at UCL Medical School on Ischaemic Preconditioning and Adaptation to Ischaemia will take place on 12 December 1995 in London. For further information please contact Jan Wenley, Symposium Administrator, Department of Molecular Pathology, 46 Cleveland Street, London W1P 6DB (tel: +44 171380 9343; fax: +44 1713873310 ). 